\title{
RESEARCH
}

\section{COMPLIANCE WITH CPAP THERAPY IN OLDER MEN WITH OBSTRUCTIVE SLEEP APNEA SYNDROME}

Turkish Journal of Geriatrics

DOI: 10.31086/tjgeri.2021.212

2021; 24(2): 165-173

- Gülay DAŞDEMIR ILKHAN ${ }^{1}$

- Hakan ÇELIKHISAR ${ }^{2}$

- Aslı KILAVUZ ${ }^{2}$

CORRESPONDANCE

${ }^{1}$ Aslı KILAVUZ

Ege University Faculty of Medicine, Division of

Geriatric Medicine, Department of Internal

Medicine, Izmir, Turkey

\section{Phone: +905323536570}

e-mail: asli.kilavuz@gmail.com

Received: Apr 05, 2021

Accepted: May 20, 2021

${ }^{1}$ Tire Public Hospital, Department of Chest Diseases, Izmir, Turkey

2 Izmir Metropolitan Municipality Hospital,

Department of Chest Diseases, Izmir, Turkey

${ }^{3}$ Ege University Faculty of Medicine, Division of Geriatric Medicine, Department of Internal Medicine, Izmir, Turkey

\section{Abstract}

Introduction: The aim of our study is to ascertain the factors affecting the compliance of older male patients with obstructive sleep apnea syndrome to continuous positive airway pressure treatment.

Materials and Method: Retrospective analysis was done on tests of older male patients who have underwent continuous positive airway pressure treatment for obstructive sleep apnea syndrome. The tests were documented for almost five years and test results of the patients were taken from records of two different hospitals. Compliance with continuous positive airway pressure treatment; using an average of 5 hours or more of continuous positive airway pressure per night was accepted.

Results: It was found that 160 of 264 older men patients adapted to continuous positive airway pressure treatment but 104 of them did not. $68 \pm$ 3.1 years were recorded as compliant group mean, while $74 \pm 4.2$ years was recorded as mean of non-compliant group. Resolution of symptoms a score of $95 \%$ were transpire in compliant patients and $87 \%$ seen in non-compliant patients. While $95 \%$ of the compliant patients took part in the continuous positive airway pressure training meetings, only $53 \%$ of the non-compliant patients attended the training meetings. A statistically significant correlation was found between non-compliance with continuous positive airway pressure therapy and smoking, nocturia, and benign prostatic hyperplasia.

Conclusion: Symptoms resolution treatment seem has linkage with improved compliance. In older male with obstructive sleep apnea syndrome, compliance with continuous positive airway pressure therapy is associated with the patient's participation in the therapy education group.

Keywords: Continuous Positive Airway Pressure; Sleep Apnea, Obstructive; Compliance; Aged 


\section{INTRODUCTION}

Obstructive sleep apnea syndrome (OSAS) refers to a sleep-associated problem that is common in people of different ages: it is identified with irregular upper airway obstruction during sleep. Usually, patients with this problem have a continuous history of apnea, hypopnea, oxygen desaturation, and disturbed sleep. Globally, OSAS is known to be a major problem affecting many people. The usual signs and symptoms are persistent snoring, frequent night awakenings, restless sleep, and immoderate daytime sleepiness (IDS). Age, gender, obesity, and nasal congestion are among the prominent risk factors associated with OSAS (1). Sleep disturbances are more prevalent among overweight people, in children, and in women in the postmenopausal period (2). They are frequently related to comorbidities such as insulin resistance, metabolic syndrome, diabetes mellitus, high blood pressure, stroke, and heart and blood vessel disease, with an increased risk of vehicle accidents and various psychiatric disorders. The management of OSAS requires persistence and an integrative approach. After the diagnosis is made, some patients are offered a consultancy service to help them cope with their illness, including their comorbidities. Recently, awareness has increased of the importance of sleep for overall life quality, as associated with well-being, particularly in older adults. Sleep architecture differs in older adults. Older adults spend more time in the shallow stages of sleep, experiencing frequent awakenings and sleep interruptions (3). However, despite numerous studies and increased awareness of sleep issues, there is no specified and systematic plan regarding the diagnosis or therapy associated with disordered breathing during sleep in older adults.

Continual positive airway pressure (CPAP) is a safe and effective therapy for patients suffering from OSAS. Properly used, CPAP is effective in reducing nocturnal respiratory events and in improving daytime sleepiness and cognitive function (4). However, it is reported that a long-term lack of adherence to treatment can reduce the compliance rate to $63 \%$ (5). Studies have shown that reduced CPAP compliance is associated with increased daytime sleepiness, a high apnea-hypopnea index (AHI), and a low mean oxygen saturation at night $(4,5)$. Individuals who have had palatal surgery report mask problems with CPAP, and this has a negative effect on compliance (6). Studies indicate that regular and uninterrupted use of CPAP therapy, for a minimum of five hours a day, has a significant effect on a patient's sense of well-being. As yet, no single definition of what constitutes good CPAP compliance has been reached, and various healthcare systems suggest different requirements. A widely accepted scale for compliance is the application of CPAP for more than five hours per night on a minimum of $70 \%$ of nights. Despite the high efficacy of CPAP therapy, its potency is restricted by variable adherence to the treatment (7). Failure to comply with CPAP treatment happens in up to $25 \%$ to $50 \%$ of patients, who often abandon the treatment within four weeks of starting it (7). In considering compliance with CPAP, the research includes adults of a wide variety of ages. However, it is known that the prevalence of OSAS increases with age, and $30 \%$ of OSAS occurs in the older population (8). Older adults thus make up a high proportion of OSAS clients. It should be kept in mind that treatment compliance factors affecting older adults may differ from the factors affecting the general population. This research considered the clinical and other variables affecting compliance with CPAP in older men (aged 65 years and above). A number of factors were ascertained to detect whether they affect compliance with CPAP among these older men. The variables included factors previously reported as affecting compliance with CPAP use. These factors may be related to the aging process and thus affect compliance indirectly.

\section{MATERIALS AND METHOD}

This research was conducted between March 2014 and April 2019 as a retrospective review of medi- 
cal records and sleep analysis of 264 male patients, aged 65 years and above who have underwent continuous positive airway pressure therapy for OSAS. The tests were documented for almost five years and test results of the patients were taken from records of two different hospitals.

Out of 320 older male patients who underwent CPAP for OSAS therapy, 264 were included in this research. Twelve patients were rejected from the study because their medical records were not comprehensive, four patients were excluded because their CPAP device lacked an hour meter, and twelve patients due to missing CPAP data. Twenty eight patients who had undergone surgery with uvulopalatopharyngoplasty and bariatric surgical methods were also excluded from the study.

All patients' medical records were reviewed for age, gender, race, AHI, CPAP pressure, body mass index (BMI), lowest oxygen saturation, tobacco and alcohol use, use of oxygen with CPAP, duration of CPAP therapy, visual and hearing impairments, CPAP complications, psychiatric disorders, change of mask required, current medications, and comorbid conditions. AHI, OSAS symptoms, nocturia, and CPAP treatment were also evaluated.

Nocturia was defined as the frequency of urination from sleep at which patients would complain to healthcare professionals. Benign prostatic hyperplasia (BPH) was diagnosed and recorded following examination by an urologist.

CPAP compliance was equated with the patient applying CPAP for five hours or more per night. All patients included in the research received at least six months of CPAP therapy. Evaluation of compliance with CPAP was calculated from recorded readings on the patient's device over the previous six months.

The term AHI refers to the sum of all apneas and hypopneas divided by the period of hours of sleep (1). Apnea refers to air flow termination of more than ten seconds. Hypopnea refers to airflow retardation, which has an effect on arterial oxygen saturation, causing desaturation of more than 3\%. Within the first month after diagnosis of OSAS and starting CPAP therapy, all patients were examined to document any resolution of sleep abnormalities, a minimum oxygen saturation of 90 and an $\mathrm{AHI}$ of less than 5. CPAP titration was applied with increasing pressures of $2.5 \mathrm{~cm}-\mathrm{H} 2 \mathrm{O}$ until the respiratory events resolved to the patients detect with polysomnography. A mask and CPAP were prescribed for all patients by a pulmonologist during the initial diagnosis. During the verification process, all patients were informed about OSAS and about the use of the CPAP mask and machine by a sleep technician. The patients were encouraged to participate in the monthly training provided in a CPAP education group led by a trained sleep technician. Three questions were asked to ascertain the patients' clinical symptoms, complications, and level of comfort with the CPAP device. A check of the equipment and its function was conducted, and any disposable items, such as masks, were changed if necessary. Meter readings were taken from the records at the same time. Patients' questions were answered, and they were allowed to share their experiences. The training sessions often included a lesson on sleep disorders. All patients were invited to the CPAP training group every six months regardless of their previous attendance.

The study had been reviewed and approved by a certified Ethical Committee, and the number of the approval documents included the date of the approval. The registration number is 2011 - KAEK-42 2019/12-05.

\section{Statistical analysis}

Fisher's exact test and the paired two-tailed Student's t-test were used for the statistical analysis. Descriptive statistics and the chi-square test were used to evaluate the data, and the within-group difference was analyzed using the Mann-Whitney $U$ 
test. Data were expressed as means $\pm \mathrm{SD}$. A statistical difference was taken as $p<0.05$.

\section{RESULTS}

The demographic and clinical characteristics of 264 older male patients with OSAS included in this study are presented in Tables 1 and 2. All patients were white Caucasian males, with mean age of 73 \pm 5.6 years during the period when the records from their CPAP devices were reviewed. All were typical OSAS patients, without respiratory failure, neuromuscular disease, or severe kyphosis or scoliosis. While 160 of the patients were compliant with the prescribed use of the CPAP device, 104 were non-compliant. As shown in Table 1, the mean age of the patients who were CPAP compliant was statistically significantly lower than that of patients who were not compliant $(p=0.03)$. When CPAP therapy was started with our patients, smoking was found to be associated with non-compliance. No statistically significant difference was shown in terms of alcohol consumption by the patients. Alcohol consumption rates were $35 \%$ in the compliant group and $60 \%$ in the non-compliant group ( $p=0.35$ ). While $95 \%$ of the compliant patients took part in the CPAP patient training group for a minimum of six months, only $53 \%$ of the non-compliant patients attended the training meetings $(p=0.006)$. Hearing loss was $9 \%$ in the non-compliant patients and $58 \%$ in the compliant patients. However, the indicated difference was statistically insignificant $(p=0.43)$. There were differences in the level of impairment in walking ability, with $8 \%$ impairment in the non-compliant group versus $26 \%$ in the compliant group ( $p=$ 0.35). This difference was statistically insignificant. No significant difference was observed between the groups in patients with visual impairment $(66 \%$ in the compliant group and $75 \%$ in the non-compliant group) $(p=0.25)$. Only $31 \%$ of the non-compli-

Table 1. Demographic and clinical characteristics of the older male patients who can comply to CPAP therapy

\begin{tabular}{|c|c|c|c|c|c|c|c|c|}
\hline $\mathbf{n}$ & $\begin{array}{c}\text { Age (Years) } \\
(\text { mean + SD) })\end{array}$ & $\begin{array}{c}\text { Age at Diagnosis } \\
\text { of OSAS } \\
\text { (Years) }\end{array}$ & $\begin{array}{c}\text { BMI } \\
\left(\mathbf{k g} / \mathbf{m}^{2}\right)\end{array}$ & $\begin{array}{c}\text { Baseline AHI } \\
\text { (events/h) }\end{array}$ & $\begin{array}{c}\text { AHI } \\
\text { on CPAP }\end{array}$ & $\begin{array}{c}\text { CPAP device } \\
\text { Pressure } \\
(\mathbf{c m}-\mathrm{H} 20)\end{array}$ & $\begin{array}{c}\text { CPAP education } \\
\text { Group Attendance } \\
(\mathbf{n}, \%)\end{array}$ & $\begin{array}{c}\text { Counter Data } \\
\text { (h/night) }\end{array}$ \\
\hline 160 & $72 \pm 2.9$ & $* 68 \pm 3.1$ & $36 \pm 12.4$ & $41 \pm 6$ & $3 \pm 2.1$ & $11 \pm 2.1$ & ${ }^{* *} 152,95 \%$ & $7.5 \pm 1.2$ \\
\hline
\end{tabular}

${ }^{*}$ Compared with the non-compliant group $p=0.03$, Student t-test. ${ }^{* *} p=0.006$, Fisher's Exact Test compared to non- compliant group. $\mathrm{BMI}$, body mass index; CPAP, continuous positive airway pressure; $\mathrm{AHI}$, Apnea-hypopnea index.

Table 2. Demographic and clinical characteristics of the older male patients who cannot comply to CPAP therapy

\begin{tabular}{|c|c|c|c|c|c|c|c|c|}
\hline $\mathbf{n}$ & $\begin{array}{c}\text { Age (Years) } \\
(\text { mean + SD) })\end{array}$ & $\begin{array}{c}\text { Age at Diagnosis } \\
\text { of OSAS } \\
(\text { Years })\end{array}$ & $\begin{array}{c}\text { BMI } \\
\left(\mathbf{k g} / \mathbf{m}^{2}\right)\end{array}$ & $\begin{array}{c}\text { Baseline AHI } \\
\text { (events/h) }\end{array}$ & $\begin{array}{c}\text { AHI } \\
\text { on CPAP }\end{array}$ & $\begin{array}{c}\text { CPAP device } \\
\text { Pressure } \\
(\mathbf{c m}-\mathrm{H} 2 \mathrm{O})\end{array}$ & $\begin{array}{c}\text { CPAP education } \\
\text { Group Attendance } \\
(\mathbf{n}, \%)\end{array}$ & $\begin{array}{c}\text { Counter Data } \\
(\mathbf{h} / \mathbf{n i g h t})\end{array}$ \\
\hline 104 & $76 \pm 3.6$ & ${ }^{*} 74 \pm 4.2$ & $34 \pm 13.1$ & $26 \pm 5$ & $5 \pm 2.4$ & $11 \pm 1.4$ & ${ }^{* *} 55,53 \%$ & $0.4 \pm 0.3$ \\
\hline
\end{tabular}

${ }^{*}$ Compared with the compliant group $p=0.03$, Student's t test. ${ }^{* *} \mathrm{p}=0.006$, Fisher's Exact Test, Compared with compliant group. BMI, body mass index; CPAP, Continuous positive airway pressure; AHI, Apnea-hypopnea index. 
ant group and $56 \%$ of the compliant group had a previous diagnosis of a psychiatric illness, but this difference was statistically insignificant $(p=0.42)$.

On average, the compliant patients had been using the CPAP for a longer time than the non-compliant patients when the records were reviewed (3.5 \pm 0.65 years, $2.4 \pm 0.64$ years, respectively; $P=0.24$ ). However, this difference is statistically insignificant. The incidence of complications from the use of the CPAP mask tended to be higher in the non-compliant patients. The impediment grade was $41 \%$ in non-compliant patients in contrast with $10 \%$ in compliant patients $(p=0.07)$, but results obtained from the report reveal that in both groups the complication rate was low. As presented in Table 3, the impediments most frequently seen were mask-related nasal problems.

Complaints of nocturia were higher in the non-compliant group than in the compliant group (82\%, 33\%, respectively; $p=0.02$ ). Remarkably, diuretic medicine was being used by many more patients in the compliance group than in the non-compliant group. Therefore, complaints of nocturia frequency from the non-compliant patients were not linked with diuretic usage.

An urology specialist physician diagnosed BPH in a significant number of non-compliant patients than in the compliant group $(62 \%, 15 \%$, respectively; $p=0.004)$. However, the available medical records did not indicate the number of night awakenings to urinate.

The baseline AHI value was higher in the CPAP compliant group than the CPAP non-compliant group ( $41 \pm 6,26 \pm 5$, respectively; $p=0.08$ ). When the initial sleep research was done, the lowest oxygen saturation recorded for the compliant group was $76 \% \pm 5 \%$, and in the non-compliant group it was $70 \% \pm 2 \%$; however, the difference between them was statistically insignificant $(p=0.25)$. No statistically significant difference was detected in the mean CPAP pressure required for adequate therapy of the two OSAS groups $(p=0.40)$. While $20 \%$ of the compliant patients needed supplemental oxygen with their CPAP therapy, 8\% of the non-compliant patients required additional oxygen, and there was no statistically significant difference $(p=0.44)$.

Early improvement of symptoms and resolution of symptoms with CPAP therapy was achieved for

Table 3. Complications of CPAP therapy in older male patients with OSAS

\begin{tabular}{|c|c|c|c|c|}
\hline Side effect & Comr & $\begin{array}{l}\text { group } \\
\text { 6) }\end{array}$ & Non-co & $\begin{array}{l}\text { ant group } \\
\text { o) }\end{array}$ \\
\hline Nasal congestion & 0 & (0) & 16 & (17) \\
\hline Skin irritation & 0 & (0) & 8 & (8) \\
\hline Mouth dryness & 8 & (5) & 0 & (0) \\
\hline Nose dryness & 0 & (0) & 8 & (8) \\
\hline Ear irritation & 8 & (5) & 0 & $(0)$ \\
\hline Cold nose & 0 & (0) & 8 & (8) \\
\hline
\end{tabular}


152 of the 160 compliant patients and 55 of the 104 non-compliant patients. The usual symptoms reported in both groups were excessive daytime sleepiness, snoring, and sleep obstruction. Excessive daytime sleepiness refers to feeling sleepy during the day. Sleep disturbance was indicated by awakening at night when the patients felt disturbed during their sleep. While an $95 \%$ resolution occurred in the OSAS symptoms in the compliant CPAP user patients, only $84 \%$ of the patients in the non-compliant group experienced symptom improvement $(p=0.002)$. Excessive daytime sleepiness and sleep disturbances were resolved in $100 \%$ of the compliant patients. However, excessive daytime sleepiness and sleep disturbance improved significantly less in the non-compliant group ( $p=0.0001, p=0.04$, respectively). Statistically significant variance was not found between the two groups in their mean AHI, as measured during the first CPAP test, in contrast to their overall symptom response $(p=0.08)$.

\section{DISCUSSION}

The research analyzed compliance with CPAP therapy in older male patients with OSAS. Patients selected were males of aged 65 years and above. We measured their compliance with treatment objectively, using the time meter.

Different clinical, behavioral, and functional variables that have a relationship with CPAP device compliance were examined. While some complaints that are encountered infrequently, such as painful eyes, were mentioned in previous studies on general CPAP compliance, due to the retrospective nature of the research, we evaluated only those factors that have been reported as affecting compliance, as these were included in the medical records. The design of this study was subject to certain limitations, and the retrospective design may be a limitation of our study. Furthermore, various comparisons were made, despite the probability that certain factors that were only randomly associated could be excluded (for example, the presence of $\mathrm{BPH}$ and age at diagnosis of OSAS). As a result of the limited number of patients, the research showed a tendency to arrive at statistical significance when comparing variables. Mean age at detection of OSAS indicated a significant difference between the compliant and non-compliant groups. The compliant patients were younger when their OSAS was first detected and their CPAP therapy started. Advancing age in the elderly population affected compliance with CPAP therapy. Certain conditions associated with advancing age affected the use of CPAP, such as cognitive failure and loss of physical ability. Further research should focus on cognitive and physical functioning in elderly patients with OSAS as this could identify factors that may restrict CPAP compliance.

Some studies have suggested that smokers are less compliant with medication than non-smokers (9). When CPAP therapy was started with our patients, smoking was found to be associated with non-compliance, while alcohol intake had no effect on differences relating to CPAP compliance. However, the value of this information is limited due to a lack of information regarding the quantity of alcohol drunk. Larger, prospective studies investigating tobacco and alcohol use in relation to CPAP compliance may determine the impact of these habits on OSAS patients.

The research noticed significant compliance improvement in patients who attended the CPAP education programme. This result is consistent with the results of a similar study investigating CPAP compliance and involving patients of various ages (10). Significant compliance variance can be detected in patient groups that undergo the education program with sleep technicians or with clinicians who are aware of the CPAP devices (11). Voluntary participation in a patient program and support group may reflect individual traits. When patients acknowledge their OSAS, their need for CPAP, and the help they derive from the program, there will be a natural tendency for both compliance and symptoms to improve. However, to ensure the conditions for the 
compliance of patients with CPAP therapy, prospective analysis would need to be applied.

Nocturia refers to awakenings at night during sleep to urinate for a minimum of two times in a total ten-hour period of sleep (12). Nocturia mostly affects older patients and is reported in $80 \%$ of adults aged 65 and above (13). Untreated OSAS was stated as a cause of nocturia in one study (14). It was described previously that $79.4 \%$ of awakenings are caused by the need to urinate when sleeping; however, the same study showed that the awakenings were actually caused by sleep apneas, hypopneas, snoring, or regular limb motion. It has been suggested that OSAS patients feel an urgent need to urinate when they are awakened during sleep by apnea or hypopnea due to an abnormal urine output pattern. It is shown that patients with OSAS have higher fractional urine streams and a lower percentage of filtered sodium reabsorption than patients who do not (15). Renal function has an effect on atrial natriuretic peptide (ANP) levels, where more negative intrathoracic pressure from respiratory effort against a closed glottis generates additional venous return and hypoxemia. As a result, hypoxemia has an effect on pulmonary vasoconstriction and right ventricular overload with right atrial distension, which is another trigger for ANP release (16). OSAS therapy with CPAP suggests a lowered urine output, but the ANP volume indicates no relationship to the extent of CPAP therapy (17). In this study, despite the application of the instruments provided, there was a rise in complaints of nocturia among the non-compliant patients; however, we discovered that the incidence of BPH detected by the physicians was significantly higher in the non-compliant group. Unless the symptoms of BPH have been resolved, the existence of a subset of OSAS patients who are less likely to follow the CPAP therapy plan must be acknowledged.

Interestingly, the incidence of diuretic use was higher in the compliant patients. There is a possibility that the use of diuretics might have an effect on the congestive heart failure therapy that is associated with sleep-disordered breathing. However, a prospective trial would be needed to authenticate or reject the clinical significance of the use of diuretics on CPAP compliance among older patients with OSAS.

It is unlikely that the factors that differ between the compliant and non-compliant groups are prostate-related, except for the age factor. In our study, we discovered that the younger patients complied better with the CPAP therapy. Statistical assessment of this possible interdependence is determined by the size of the study population. We discovered statistically insignificant variance between compliant and non-compliant patients in the baselines for $\mathrm{AHI}$, mean lowest oxygen saturation levels in the first sleep study, and prescribed CPAP pressure. Furthermore, the percentage of patients responding to CPAP therapy rose significantly in the compliant group. This is in uniformity with other research in the literature (18-20). It can be considered that the resolution of symptoms is the primary reason for patients continuing with such a potentially disturbing treatment. Problems with the CPAP mask increase the risk of non-compliance. Therefore, it is vital for patients to maintain a CPAP pressure that effectively treats their OASA so that compliance with CPAP and symptom resolution will increase. For this reason, impediments produced by the mask need to be ascertained and redressed in CPAP immediately. In addition, it is important for the elderly with OSAS to access a trained healthcare technician with knowledge of CPAP devices in order to be equipped with a suitable apparatus that will ensure their comfort.

\section{CONCLUSION}

The CPAP records for the last 6 months of older men with OSAS were examined to ascertain factors that linked with treatment compliance. Compliant patients were, on average, younger than non-compliant patients when their OSAS was diagnosed, and they participated better in the CPAP patient 
training group. Complete resolution of OSAS symptoms through the use of CPAP happened more frequently in the compliant patient group. Non-compliant patients smoked more, complained more of nocturia, were more often diagnosed with $\mathrm{BPH}$, and experienced more side effects from the CPAP

\section{REFERENCES}

1. M Sateia. Sleep Related Breathing Disorders, In: M Sateia (Ed). International Classification of Sleep Disorders, 3th edition, American Academy of Sleep Medicine, USA 2014, pp 49-141.

2. Yılmaz Durmaz $D$, Güneş $A$. Which is more important: the number or duration of respiratory events to determine the severity of obstructive sleep apnea? Aging Male 2020; 23 (2): 119-24. (PMID: 31241414).

3. Cao MT, Sternbach JM, Guilleminault C. Continuous positive airway pressure therapy in obstuctive sleep apnea: benefits and alternatives. Expert Rev Respir Med 2017; 11 (4): 259-72. (PMID: 28287009).

4. Lin J, Suurna M. Sleep Apnea and Sleep-Disordered Breathing. Otolaryngol Clin North Am 2018; 51 (4): 827-33. (PMID: 29779616).

5. Patil SP, Ayappa IA, Caples SM, Kimoff RJ, Patel SR, Harrod CG. Treatment of Adult Obstructive Sleep Apnea With Positive Airway Pressure: An American Academy of Sleep Medicine Systematic Review, Meta-Analysis, and GRADE Assessment. J Clin Sleep Med 2019; 15 (2): 301-34. (PMID: 30736888).

6. Semelka M, Wilson J, Floyd R. Diagnosis and Treatment of Obstructive Sleep Apnea in Adults. Am Fam Physician 2016; 94 (5): 355-60. (PMID: 27583421).

7. Singam $P$, Hong $G E$, Ho $C$, Hee TG, Jasman $H$, Inn FX, Bahadzor B, Tamil A, Zainuddin Z. Nocturia in patients with benign prostatic hyperplasia: evaluating the significance of ageing, co-morbid illnesses, lifestyle and medical therapy in treatment outcome in real life practice. Aging Male 2015; 18 (2): 112-7. (PMID: 25690022).

8. Leng Y, McEvoy CT, Allen IE, Yaffe K. Association of Sleep-Disordered Breathing With Cognitive Function and Risk of Cognitive Impairment: A Systematic Review and Meta-analysis. JAMA Neurol 2017; 74 mask. Some of these factors, particularly BPH, are peculiar to older men with OSAS. To validate our study, a huge prospective trial would be required. Additional research is needed to contrast the rates of compliance and the factors affecting compliance between young and old patients.

(10): 1237-45. Erratum in: JAMA Neurol 2018; 75 (1): 133. (PMID: 28846764).

9. Berlowitz DR, Foy CG, Kazis LE et al. Effect of Intensive Blood-Pressure Treatment on Patient-Reported Outcomes. N Engl J Med 2017; 377 (8): 733-44. (PMID: 28834483).

10. Pien GW, Ye L, Keenan BT, Maislin G et al. Changing Faces of Obstructive Sleep Apnea: Treatment Effects by Cluster Designation in the Icelandic Sleep Apnea Cohort. Sleep 2018; 41 (3): zsx201. (PMID: 29301021).

11. Deering $S$, Liu L, Zamora T, Hamilton J, Stepnowsky C. CPAP Adherence is Associated With Attentional Improvements in a Group of Primarily Male Patients With Moderate to Severe OSA. J Clin Sleep Med 2017; 13 (12): 1423-8. (PMID: 29065955).

12. Zhu H, Xu H, Chen $R$ et al. Smoking, obstructive sleep apnea syndrome and their combined effects on metabolic parameters: Evidence from a large cross-sectional study. Sci Rep 2017; 7 (1): 8851. (PMID: 28821856).

13. Pelosi LB, Silveira MLC, Eckeli AL et al. The importance of clinical monitoring for compliance with Continuous Positive Airway Pressure. Braz J Otorhinolaryngol 2017; 83 (4): 439-44. (PMID: 27472984).

14. Shigehara K, Konaka H, Sugimoto K et al. Sleep disturbance as a clinical sign for severe hypogonadism: efficacy of testosterone replacement therapy on sleep disturbance among hypogonadal men without obstructive sleep apnea. Aging Male 2018; 21 (2): 99105. (PMID: 28920756).

15. Balodiya S, Kamath A. Evaluation of Hospital Discharge Prescriptions in the Elderly and Younger Adults Using the Medication Regimen Complexity Index. Curr Drug Saf 2019; 14 (2): 116-21. (PMID: 30523768).

16. Benjafield AV, Pépin JL, Valentine $\mathrm{K}$ et al. Compli- 
ance after switching from CPAP to bilevel for patients with non-compliant OSA: big data analysis. BMJ Open Respir Res 2019; 6 (1): e000380. (PMID: 31178999).

17. Brunello A, Ahcene-Djaballah S, Lettiero A et al. Prevalence of pain in patients with cancer aged 70 years or older: A prospective observational study. J Geriatr Oncol 2019; 10 (4): 637-42. (PMID: 30686679).

18. Loffler KA, Heeley E, Freed R et al. Effect of Obstructive Sleep Apnea Treatment on Renal Function in $\mathrm{Pa}$ - tients with Cardiovascular Disease. Am J Respir Crit Care Med 2017; 196 (11): 1456-62. (PMID: 28743190).

19. Mclnnis RP, Dodds EB, Johnsen J, Auerbach S, Pyatkevich Y. CPAP Treats Enuresis in Adults With Obstructive Sleep Apnea. J Clin Sleep Med 2017; 13 (10): 1209-12. (PMID: 28859721).

20. Monaghan TF, Epstein MR, Bliwise DL et al. Time of peak nocturnal diuresis rate between men with secondary nocturnal polyuria versus nocturnal polyuria syndrome. Neurourol Urodyn 2020; 39 (2): 785-92. (PMID: 31961968). 